\title{
Asian Clinical Symposium
}

July 20, 2012

The 44th Annual Scientific Meeting of the Japan Atherosclerosis Society in Fukuoka, Japan

\section{Regional Differences in Atherosclerotic Risk Factors and Cardiovascular Outcomes in Asian} Population

\author{
Moderators : Chee Eng Tan \\ Gleneagles Medical Centre, Singapore \\ Brian Tomlinson \\ Department of Medicine and Therapeutics, the Chinese University of Hong Kong \\ Wei-Hsian Yin \\ Cheng Hsin General Hospital, Taiwan \\ Shizuya Yamashita \\ Department of Cardiovascular Medicine, Osaka University Graduate School of Medicine \\ Jaw-Wen Chen \\ Cardiology, Taipei Veterans General Hospital
}

1. Explanation for the Japanese paradox: prevention of increase in coronary heart disease and reduction in stroke

Hirotsugu Ueshima

Department of Health Science, Shiga University of Medical Science

2. Trends in the incidence and mortality of stroke and myocardial infarction in Asian Pacific region-The Singapore Experience

\section{E Shyong Tai}

Singapore Health Services

3. Metabolic risk factor profile in Southeast Asia

Rody G. Sy

Section of Cardiology, Department of Medicine, University of the Philippines College of Medicine

4. Arterial stiffness contributes to coronary artery disease risk prediction beyond the traditional risk score (RAMA-EGAT score)

Teerapat Yingchoncharoen ${ }^{1}$ Thosaphol Limpijankit ${ }^{1}$ Sutipong Jongjirasiri² Jiraporn Laothammatas 2

Sukit Yamwong ${ }^{1}$ P Piyamitr Sritara ${ }^{1}$

${ }^{1}$ Division of Cardiology, Department of Medicine, Faculty of Medicine, Ramathibodi Hospital, Mahidol University, Bangkok, Thailand

${ }^{2}$ Department of Radiology, Faculty of Medicine, Ramathibodi Hospital, Mahidol University, Bangkok, Thailand

5. Gender difference in factors associated with cardiovascular and cerebrovascular disease

\section{Hung-I Yeh}

Department of Medicine, Mackay Medical College, Taiwan

6. Risk factors of cardiovascular disease in china

Chongyou Lee

Cardiology, Peking University People’ s Hospital, Beijing, China 


\section{Asian Clinical Symposium}

7. Complication of Acute Myocardial Infarction in Relation with Major Risk Factors

Djoko Soemantri

Department of Cardiology Faculty of Medicine, Airlangga University/Dr. Soetomo General Hospital Surabaya, Indonesia

8. Metabolic Syndrome and Cardiovascular Diseases

Moon-Kyu Lee

Division of Endocrinology \& Metabolism, Department of Medicine Samsung Medical Center, Sungkyunkwan University School of Medicine, Seoul, Korea

9. The risk factors in acute coronary syndrome in patients over 65 years old at Thong Nhat hospital of Ho Chi Minh city, Vietnam

Nguyen Duc Cong, Ho Thuong Dung

Thong Nhat Hospiatl of Ho Chi Minh city, Vietnam

Discussion

Moderators : Shizuya Yamashita

Department of Cardiovascular Medicine, Osaka University Graduate School of Medicine

Kensuke Egashira

Department of Cardiovascular Medicine, Graduate School of Medical Sciences, Kyusyu University 